\title{
A THEOREM ON NILPOTENT GROUPS WITH RESTRICTED EMBEDDINGS
}

\author{
KARL H. WEHRHAHN
}

\begin{abstract}
Suppose that $G$ is a nonabelian group with a unique proper normal subgroup of some given order. It is proved that $G$ is not contained as a normal subgroup within the Frattini subgroup of a finite $p$-group.
\end{abstract}

Burnside [1] has proved that nonabelian groups with cyclic center, or whose derived subgroup has index $p^{2}$ cannot occur as the derived subgroup of a $p$-group. Hobby [2] showed that the same classes of groups also cannot occur as the Frattini subgroup of a $p$-group. Nonabelian $p$-groups with cyclic center or whose derived subgroup has index $p^{2}$ are examples of groups which have a unique proper normal subgroup of some given order. In this note we prove that the theorems of Burnside and Hobby may be generalized to include this wider class of $p$-groups. In fact we prove the following more general theorem. ${ }^{1}$

TheOREM. Suppose $G$ is a nonabelian group with a unique proper normal subgroup of some given order. Then $G$ is not contained as a normal subgroup within the Frattini subgroup of a finite p-group.

Proof. Denote by $G_{0}$ the unique proper normal subgroup of $G$. Suppose first that $G_{0}$ is cyclic and that $G$ is a normal subgroup of a $p$-group $P$ within the Frattini subgroup $\Phi(P)$. By [3, Satz III 7.5] there exists an elementary abelian subgroup $R$ of $G$ which is normal in $P$ and has type $(p, p)$. Since $G_{0}$ is cyclic it is contained in $R$ and so has order $p$. As $G_{0}$ is the only normal subgroup of $G$ having order $p$, it follows that $Z(G)$ is cyclic. However, since $R$ is normal in $P$, the order of $P / C_{P}(R)$ divides the order of the group of automorphisms of $R$. Hence, $C_{P}(R)$ is either $P$ itself or a maximal subgroup of $P$.

In either case $G \leqslant \Phi(P) \leqslant C_{P}(R)$ and, hence, $R \leqslant Z(G)$ contrary to the fact that $Z(G)$ is cyclic. Thus the theorem holds if $G_{0}$ is cyclic.

To complete the proof we use induction on the order of $G_{0}$. Suppose $G_{0}$ is noncyclic and $G$ is a normal subgroup of a $p$-group $P$ within the Frattini subgroup $\Phi(P)$. Let $L$ be a normal subgroup of $P$ of order $p$ contained in $G_{0}$; then $G_{0} / L$ is a unique proper normal subgroup of $G / L$ of the given order $\left|G_{0} / L\right|<\left|G_{0}\right|$. Also $G / L \leqslant \Phi(P) / L=\Phi(P / L)$ and by induction $G / L$ is abelian and, hence, cyclic. Since $L \leqslant Z(G), G$ is abelian, contrary to the hypothesis of the theorem. This completes the proof of the theorem.

Received by the editors January 17, 1975.

AMS (MOS) subject classifications (1970). Primary 20Dxx, $20 \mathrm{D} 15$.

${ }^{1} \mathrm{I}$ am indebted to the referee whose comments led to the present generalization. 


\section{REFERENCES}

1. W. Burnside, On some properties of groups whose orders are powers of primes, Proc. London Math. Soc. (2) 11(1912), 225-245.

2. C. R. Hobby, The Frattini subgroup of a p-group, Pacific J. Math. 10(1960), 209-212. MR22 \#4780.

3. B. Huppert, Endliche Gruppen. I, Die Grundlehren der math. Wissenschaften, Band 134, Springer-Verlag, Berlin and New York, 1967. MR37 \#302.

Department of Mathematics, The University of Calgary, Calgary, Alberta, T2N 1N4, CANADA 